Nous sommes au vingt-et-unième siècle. Tous les changements d'état de la matière sont décrits par la théorie des transitions de phases. Tous ? Non.

Un phénomène résiste encore et toujours aux investigations théoriques : la transition vitreuse.

\section{De surcroît, cette dernière} semble modifiée dans les films minces de polymères, ce qui pourrait d'une part nous renseigner sur ses mécanismes intimes, et d'autre part remettre en cause l'utilisation de ces films au travers d'applications prometteuses. II apparaît donc essentiel de mesurer et comprendre les effets du confinement et des interfaces sur la transition vitreuse.

\section{Remerciements}

Ces résultats sont le fruit d'une collaboration internationale et interdisciplinaire incluant : James Forrest (Perimeter Institute et University of Waterloo, Canada), Kari Dalnoki-Veress (McMaster University, Hamilton, Canada), Yu Chai (Waterloo University, Canada), Joshua McGraw (LPS, ENS, Paris, France), Justin Salez (Université Paris Diderot, France), Oliver Bäumchen (Max Planck Institut, Göttingen, Allemagne), et Michael Benzaquen (CFM, Paris, France). Nous les remercions vivement.

\title{
La transition vitreuse aux interfaces
}

Thomas Salez (thomas.salez@espci.fr) et Élie Raphaël

Laboratoire de Physico-Chimie Théorique, UMR CNRS 7083 Gulliver, ESPCI-ParisTech, PSL Research University,

10 rue Vauquelin, 75231 Paris Cedex 05

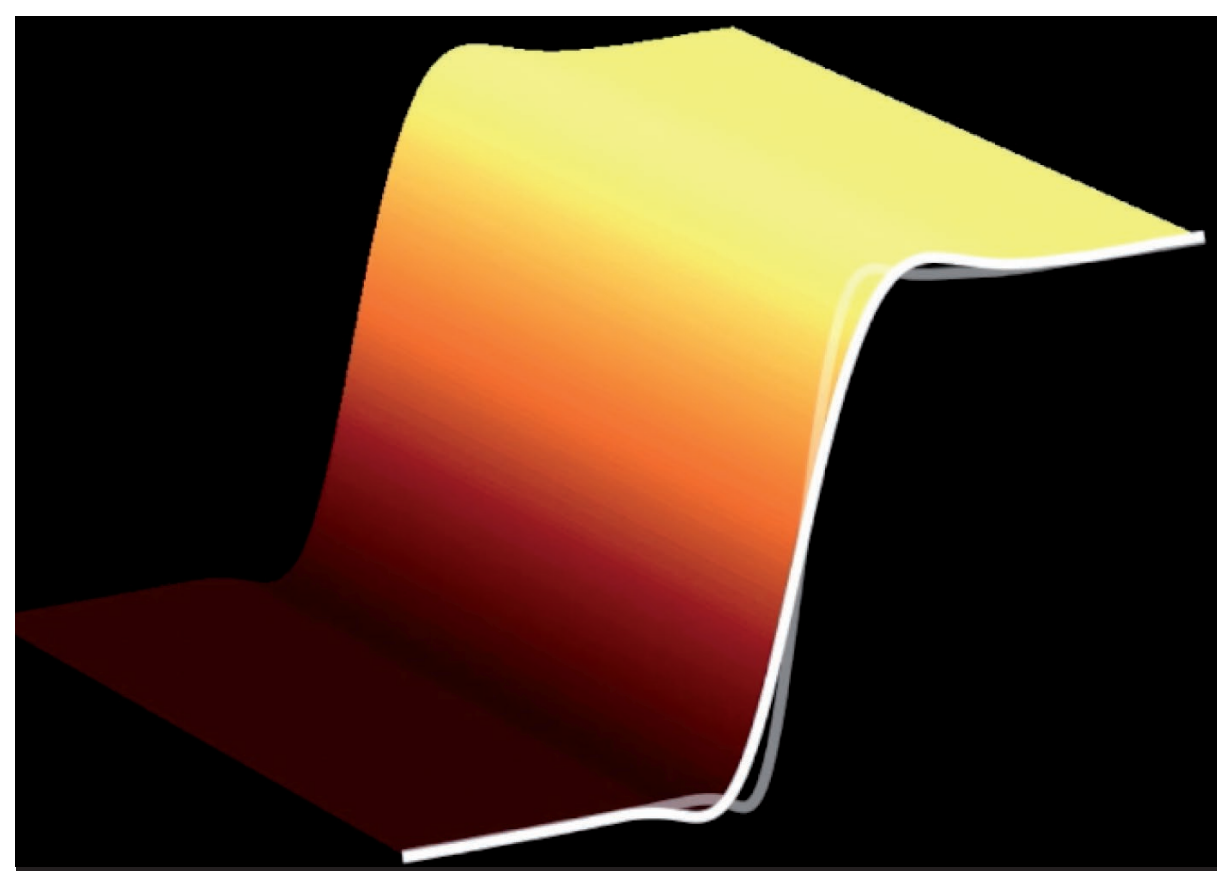

La surface d’un nanofilm vitreux relaxe dans le temps ! Image de surface (AFM, couleurs artificielles) prise au cours de la relaxation d'une nanomarche de polystyrène vitreux $\left(T=40^{\circ} \mathrm{C}=\mathrm{T}_{\mathrm{g}}-30^{\circ} \mathrm{C}\right)$, de poids moléculaire $3 \mathrm{~kg} / \mathrm{mol}$.

\section{La transition vitreuse}

Quel est le mécanisme responsable de la transition vitreuse, ce changement d'état entre un liquide visqueux et un solide amorphe obtenu par refroidissement rapide, alors même que les structures atomiques sous-jacentes paraissent peu modifiées ? Pourquoi ce phénomène physique apparemment anodin, et maitrisé par les souffleurs de verre depuis la Rome antique, se révèle-t-il si difficile à comprendre et tellement différent de la transition liquidesolide classique?

Ces questions ne sont pas nouvelles et continuent de faire couler beaucoup d'encre, compte tenu de la place occupée par les verres et les plastiques dans l'art, l'industrie et les nanotechnologies, ainsi que leur grande diversité : des verres moléculaires aux verres organiques de polymères, en passant par les verres métalliques, les verres de spins, les verres colloïdaux ou les milieux granulaires encombrés.

\section{Coopérativité et confinement}

Malgré l'absence de théorie unifiée, un concept central s'est peu à peu imposé dans les années 1960 pour décrire la dynamique des verres : la coopérativité. Dans un liquide usuel, les mouvements moléculaires ne sont pas corrélés. En revanche, au sein des matériaux vitreux, la dynamique s'effectuerait via des régions de taille $\xi$ dans lesquelles les molécules se réarrangeraient collectivement. Cette échelle de longueur de coopérativité, supposée diverger à température finie, a fait et continue de faire l'objet d'intenses recherches.

Malheureusement, il est illusoire d'espérer observer des réarrangements structurels macroscopiques dans l'état vitreux, en raison des temps mis en jeu qui sont colossaux par rapport à la durée d'une expérience usuelle. De même, il est impossible de traquer des molécules uniques en volume avec les techniques actuelles de 
microscopie. On ne peut donc pas mesurer $\xi$ dans un verre massif.

Une solution alternative a germé dans les années 1990 : fabriquer des échantillons de taille comparable à $\xi$ et observer leurs anomalies. Cependant, l'unité élémentaire de mesure de $\xi$ étant par définition une taille de molécule, il fallait pouvoir fabriquer et observer dynamiquement des échantillons stables de quelques tailles moléculaires. Tâche a priori impossible. Sauf avec les films de polymères, et ce pour trois raisons : d'une part, les macromolécules amplifient les tailles moléculaires ; d'autre part, leurs enchevêtrements conferent aux films des propriétés de stabilité remarquables, comme l'ont bien remarqué les industriels ; enfin, la fabrication des échantillons est relativement aisée par enduction centrifuge ${ }^{(1)}$.

\section{Anomalies dans les nanofilms de polymères}

Comme nombre de matériaux, un film de polymère se dilate avec la température. Cette dilatation peut être détectée et quantifiée précisément par ellipsométrie (une mesure du changement de polarisation de la lumière après réflexion sur l'échantillon). En diminuant la température, le matériau se contracte progressivement avec un coefficient de dilatation thermique constant. Puis, à une température donnée - de l'ordre d'une centaine de degrés Celsius pour le polystyrène, par exemple - la valeur numérique du coefficient de dilatation chute : c'est la transition vitreuse. Cette méthode de dilatométrie, semblable à ses consœurs telles que la calorimétrie, permet donc de mesurer la température $\mathrm{T}_{\mathrm{g}}$ de transition vitreuse.

Ainsi, plusieurs équipes sont parvenues à montrer que la température de transition vitreuse d'un film de polystyrène dépend de son épaisseur moyenne, quand cette dernière est inférieure à $50 \mathrm{~nm}$ (fig. 1). De plus, des études de dynamique locale, réalisées par exemple à l'aide de l'enfoncement de nanoparticules d'or, de l'observation de fluctuations thermiques, ou du suivi de traceurs, révèlent une seconde anomalie dans les films : l'existence d'une couche superficielle plus mobile, de quelques nanomètres d'épaisseur. Mais l'ensemble de ces observations demeure fortement controversé. L'origine et les propriétés de cette couche sont indéterminées, et le lien avec le décalage de $T_{g}$ reste hypothétique.

\section{Enjeux des films minces et de leur rhéologie}

Les microfilms et nanofilms - liquides, élastiques ou vitreux - de polymères sont d'une grande importance dans de nombreux champs scientifiques interdisciplinaires, tels que la physico-chimie, la physiologie, la biophysique, la microélectronique ou la chimie des surfaces. Outre leur intérêt sur le plan fondamental, ils entrent en jeu dans des processus industriels tant optiques que mécaniques ou chimiques, au travers de la nanolithographie, de la lubrification, des peintures, des traitements de surface et des membranes élastiques. Il en ira vraisemblablement de même dans l'avenir avec le développement de l'électronique moléculaire, des dispositifs souples d'affichage multimédia, de la biomimétique, de la superadhésion ou encore des surfaces autonettoyantes. À titre d'exemple, ces films sont des candidats potentiels pour le stockage massif de données, au travers du projet Millipede d'IBM. Ce dernier consiste à indenter des films minces de polymères à l'échelle nanométrique afin d'y graver mécaniquement de l'information, et ainsi augmenter considérablement les densités surfaciques de stockage.

$\mathrm{Au}$ travers de ces applications potentielles, on conçoit aisément qu'une compréhension fine de la physique qui régit la stabilité et la dynamique de ces systèmes est une étape importante. L'épaisseur typique (10-100 nm) des nanofilms de polymères étant comparable à la taille caractéristique des macromolécules constitutives, l'effet de confinement est susceptible d'altérer fortement les propriétés de ces dernières. La nanorhéologie de ces objets soulève donc de nombreuses questions vivement débattues à ce jour, à la frontière du continu et du discret. Outre les anomalies de transition vitreuse au cœur de cette étude, citons deux exemples. Tout d'abord, les modifications des conformations intramoléculaires et des enchevêtrements intermoléculaires aux abords des surfaces et dans des géométries confinées, pourraient affecter directement la viscosité et donc la stabilité des films. Ensuite, les phénomènes interfaciaux étant dominants dans les films minces, les propriétés de ces derniers pourraient dépendre du glissement aux bords, fortement amplifié pour des polymères enchevêtrés.

\〉

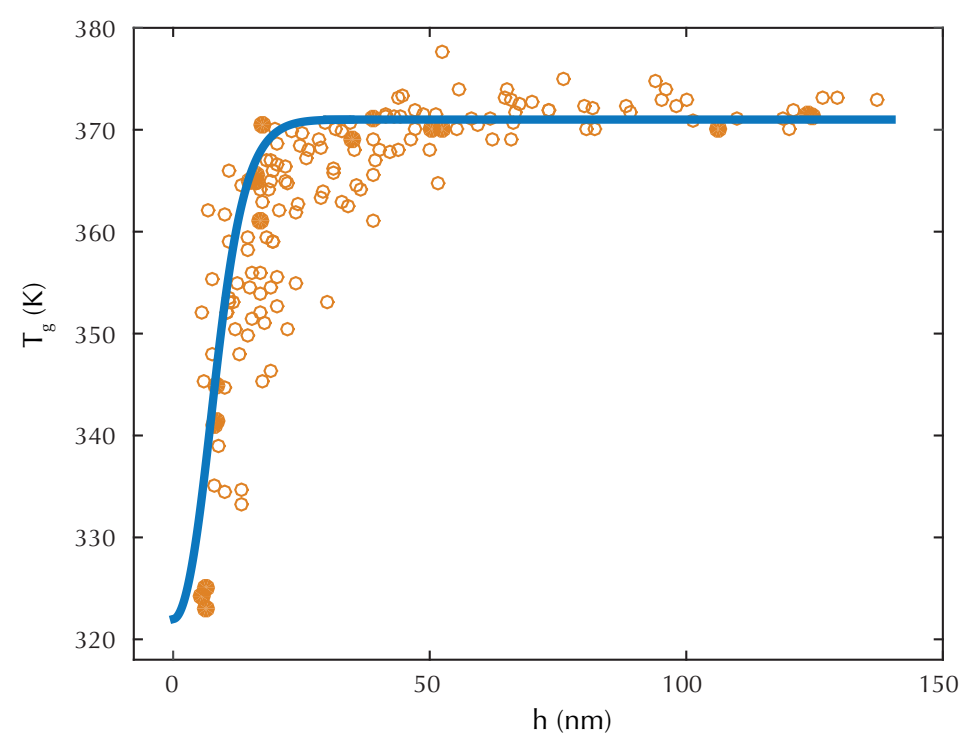

1. Position du problème. Température $T_{g}$ de transition vitreuse de films de polystyrène placés sur des substrats de silicium, mesurée par dilatométrie, en fonction de l'épaisseur h des films. Les ronds représentent les données de la littérature. L'hypothèse microscopique des chaînes coopératives aléatoires (voir fin de l'article) permet de quantifier la mobilité moléculaire locale et donc la température de transition vitreuse (trait plein). 


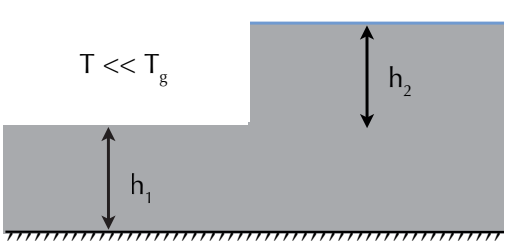

2. Système et notations. Après fabrication, l'échantillon est un film de polystyrène en forme de marche d'escalier - une nanomarche - formé de deux couches d'épaisseurs $h_{1}$ et $h_{2}$ de l'ordre de 100 nanomètres, à température ambiante $\mathrm{T}$ très inférieure à la température de transition vitreuse $T_{g}$.
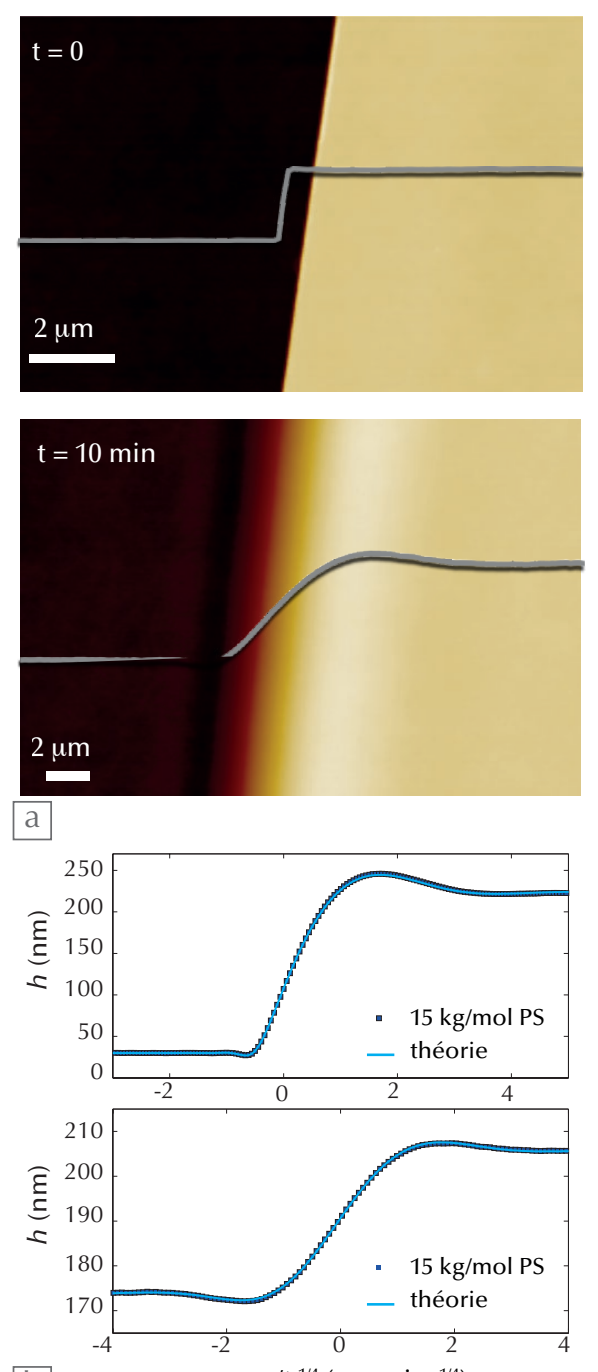

b

$\mathrm{x} / \mathrm{t}^{1 / 4}\left(\mu \mathrm{m} \min ^{-1 / 4}\right)$

3. Observations. (a) Images en microscopie à force atomique de la relaxation d'une nanomarche de polystyrène liquide $\left(T>T_{g}\right)$, de poids moléculaire $15 \mathrm{~kg} / \mathrm{mol}$, au cours du temps t. Les contraintes capillaires dans les coins de la marche sont telles que la pression de Laplace ${ }^{(2)}$ engendre un écoulement visqueux et des oscillations de surface. (b) Comparaison entre profil expérimental $h(x, t)$, où $x$ est la position horizontale, et modèle hydrodynamique, pour deux hauteurs de marche à $t=10$ min. Le seul paramètre ajustable est un facteur d'étirement horizontal directement relié à la viscosité du film liquide, et qui permet donc sa mesure précise.

\section{\〉}

\section{La méthode des nanomarches}

Afin de comprendre les anomalies de transition vitreuse dans les films minces, et plus généralement la rhéologie de ces objets, il faut une nanosonde, c'est-à-dire un dispositif permettant de mesurer les phénomènes physiques aux échelles nanométriques. Par exemple, pour comprendre si un matériau coule et quantifier son écoulement, il suffit de déformer sa surface et d'observer son évolution. C'est l'expérience que l'on fait instinctivement en perturbant la surface du miel dans un pot à l'aide d'une cuillère. La déformation s'estompe progressivement sous l'effet de la gravité qui tend à aplatir la surface. Cette relaxation s'opère sur un temps d'autant plus long que le liquide est froid, et donc visqueux. Ce temps caractéristique est une mesure directe de la viscosité du matériau.

À l'échelle nanométrique, le moteur est la tension de surface (qui contrôle par ailleurs la forme des petites gouttes d'eau et des bulles de savon) : elle l'emporte sur la gravité. Ainsi, en déformant l'interface libre d'un nanofilm de polymère placé sur un substrat rigide, et en observant son aplanissement capillaire sur plusieurs dizaines de minutes, on peut remonter à la mobilité interne du matériau [1]. Le dispositif développé consiste plus précisément à fabriquer des nanofilms de polystyrène fondu en forme de marches d'escalier (fig. 2), en superposant deux films plats d'épaisseur typique $\sim 100 \mathrm{~nm}$ et de largeurs différentes, puis à observer leur relaxation à l'aide d'un microscope à force atomique (fig. 3a). L'évolution et la forme des profils étant décrites par la mécanique des fluides, on peut ainsi calibrer cette nanosonde avec précision (fig. 3b).

\section{Mesure de la mobilité de surface}

À l'aide de la méthode des nanomarches, il est alors possible d'étudier la relaxation capillaire de films de polystyrène en dessous de leur température de transition vitreuse [2]. La conclusion est surprenante : un verre mince de polymère coule ! Cet écoulement a lieu sur quelques heures, et subsiste sur plusieurs dizaines de degrés en dessous de $\mathrm{T}_{\mathrm{g}}$, alors qu'un échantillon épais aux mêmes températures ne semble pas relaxer pendant plusieurs années. Ces observations peuvent être comprises à l'aide d'un modèle mésoscopique simple à deux couches. Sous la température de transition vitreuse, la dynamique est hétérogène et le film présente une région de surface liquide (fig. 4a) dont l'épaisseur croît et gagne tout l'échantillon (fig. 4b) lors de la transition au chauffage. Le résultat important est que la mobilité (conductance hydraulique qui relie le débit surfacique au gradient de pression dans un écoulement visqueux plan) de cette couche de surface peut être extraite (fig. 5) d'un ajustement des données expérimentales par le modèle mésoscopique (figs. 3b, 4 a et $4 b$ ).

\section{La théorie des chaînes coopératives aléatoires}

Afin de comprendre l'origine d'une telle couche liquide en surface des films vitreux, et son rôle éventuel dans les réductions de $\mathrm{T}_{\mathrm{g}}$ observées quand l'épaisseur du film diminue (fig. 1), il faut pour finir se doter d'une théorie microscopique [3]. Au sein d'un verre, les molécules sont dans un environnement analogue à celui d'un voyageur dans un métro aux heures de pointe. Chaque molécule est prisonnière d'une cage formée par ses voisines, et possède très peu de volume libre pour se déplacer, changer de place, et donc permettre la relaxation du matériau vers l'équilibre. Dans l'impossibilité de bouger individuellement, l'alternative est le mouvement coopératif : plusieurs molécules se déplacent simultanément, comme les passagers du métro lors des réarrangements provoqués par les montées et descentes aux stations. En s'appuyant sur certaines observations faites dans les milieux granulaires et dans les simulations numériques de dynamique moléculaire, on peut même supposer que ces régions coopératives prennent la forme de trains unidimensionnels de molécules, plutôt que d'amas tridimensionnels : on parle de chaînes coopératives aléatoires. À l'aide d'outils de physique statistique, il est alors possible de retrouver quantitativement la phénoménologie usuelle des verres en volume.

Enfin, on peut ajouter une interface libre à la description proposée ci-dessus. On parvient ainsi pour la première fois à expliquer de manière quantitative les deux anomalies évoquées précédemment [3]. La clé réside dans le fait qu'une chaîne coopérative aléatoire qui parvient à une interface libre est tronquée par cette dernière en raison de la disparition des contraintes d'encombrement (fig. 4c). En clair, un 
passager du métro proche de la porte n'a pas besoin de jouer collectif pour participer au réarrangement de la rame : il peut en sortir seul, comme une molécule dans un liquide. Ce type de passager fluidifie donc le voisinage de la porte. En invoquant une description brownienne des chaînes coopératives et leur probabilité de premier passage à l'interface, on parvient alors à quantifier le temps de relaxation local d'un film, et donc ses propriétés de mobilité (fig. 5) et de transition vitreuse (fig. 1).

\section{Conclusion}

Ironie du sort, l'hypothèse erronée selon laquelle les vitraux d'églises seraient déformés en raison de leur caractère liquide, se révèle être partiellement vérifiée dans les nanofilms de polymères, aux abords de leur température de transition vitreuse. Le verre de polymères - matériau incontournable de l'industrie et des nanotechnologies - est traditionnellement apparenté à un solide amorphe... et pourtant il coule ! Du moins en surface, et sur quelques nanomètres de profondeur, ce qui a pour effet de réduire sa température de transition vitreuse effective. Grâce à la méthode des nanomarches, il est maintenant possible de caractériser finement les propriétés dynamiques des couches superficielles des verres de polymères, en fonction de la température et pour des compositions variées. La méthode ne se limite d'ailleurs pas à ces matériaux et pourra plus généralement permettre de sonder la matière condensée molle, en confinement à l'échelle du nanomètre, là où les interfaces et les tailles moléculaires finies jouent un rôle prédominant.

Enfin, la théorie microscopique des chaînes coopératives aléatoires possède deux avantages : sa simplicité analytique et son caractère pictural, qui pourraient contribuer à en faire un outil pratique pour l'étude de la transition vitreuse. On peut alors espérer comprendre un peu mieux les mécanismes de ce phénomène et ses anomalies dans les films, et ainsi, par exemple, guider la fabrication de verres ultra-stables, ces nouveaux matériaux en pleine émergence.

(1) Enduction centrifuge ("spin coating") : technique de dépôt de couches minces d'épaisseur uniforme sur la surface lisse d'un substrat tournant à haute vitesse constante.

(2) Pression de Laplace : différence de pression entre deux milieux séparés par une interface courbe.

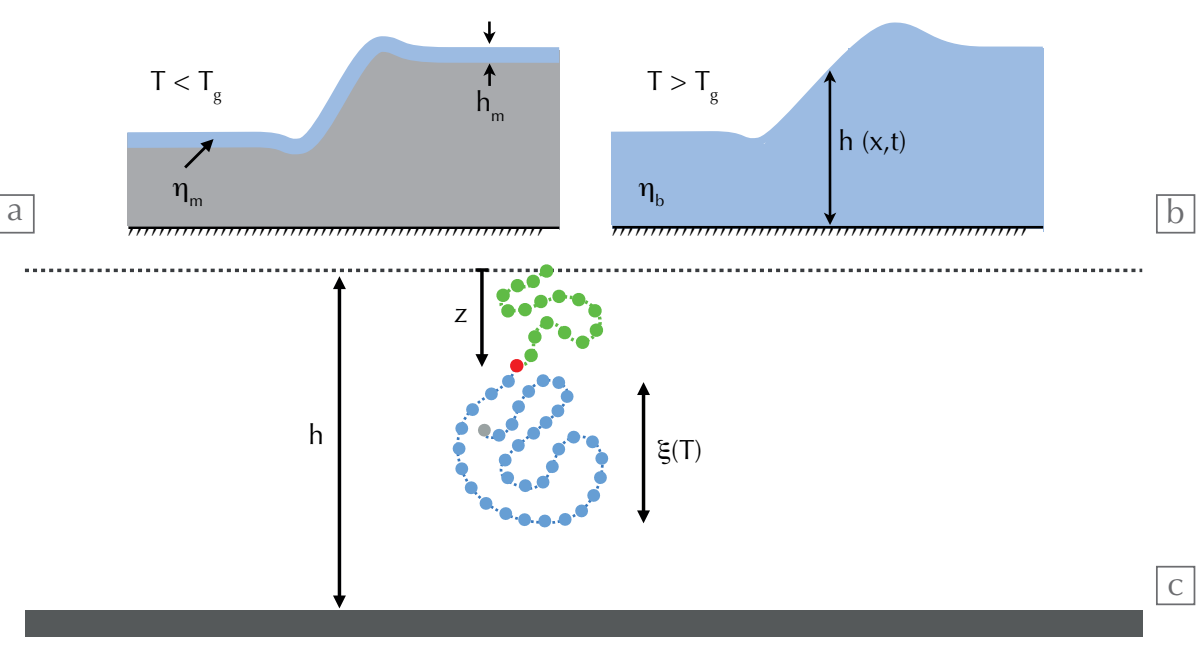

4. Modélisation. (a) (mésoscopique). Pour une gamme de températures inférieures à $T_{g}$, l'écoulement est localisé sur une couche superficielle (en bleu) d'épaisseur $h_{m}$ de l'ordre de quelques nanomètres. Au cours de l'évolution en surface, une région initialement immobile (grise) peut se retrouver à une distance inférieure à $\mathrm{h}_{\mathrm{m}}$ de l'interface libre et ainsi devenir liquide (bleue), ce qui explique également le réarrangement de la partie grise. (b) (mésoscopique). Pour une gamme de températures supérieures à $\mathrm{T}_{\mathrm{g}}$, l'écoulement s'effectue dans tout l'échantillon. (c) (microscopique). Troncature des chaînes coopératives à l'interface libre d'un film vitreux. À température T, dans un film d'épaisseur h, à distance z d'une interface libre (tirets), une molécule (rouge) peut se déplacer à l'aide d'une chaîne coopérative aléatoire (bleue) de taille $\xi$ dans le cœur du matériau, mais elle peut alternativement le faire au travers d'une chaîne tronquée (verte) par l'interface - plus courte, donc plus probable -, ce qui accroît la mobilité de surface et réduit ainsi la température de transition vitreuse du film.

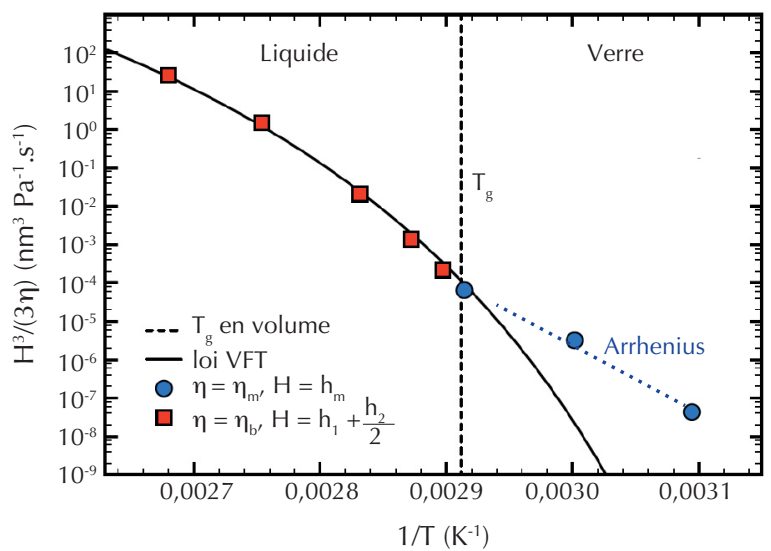

5. Mobilité $H^{3} /(3 \eta)$ d'un film de polymère (définie via l'épaisseur $H$ et la viscosité $\eta$ de la région d'écoulement) en fonction de l'inverse de la température T. Fait caractéristique de la transition vitreuse, la mobilité varie sur dix décades aux abords de $\mathrm{T}_{\mathrm{g}}$. Lorsque $\mathrm{T}>\mathrm{T}_{\mathrm{g}}$ (carrés), l'écoulement a lieu dans tout le film (fig. 4b), d'épaisseur moyenne $H=h_{1}+h_{2} / 2$ et de viscosité en volume $\eta=\eta_{b}$. Cette dernière suit la loi phénoménologique de Vogel-Fulcher-Tammann (VFT), caractéristique des liquides surfondus. Lorsque $\mathrm{T}<\mathrm{T}_{\mathrm{g}}$ (ronds), l'écoulement est localisé dans une couche de surface (fig. 4a) d'épaisseur $H=h_{m}$ et de viscosité $\eta=\eta_{m}$, qui semble cette fois suivre une loi d'Arrhenius caractéristique d'un liquide simple. La viscosité $\eta_{m}$ et l'épaisseur $h_{m}$ de la couche mobile ne sont pas déterminées de façon indépendante dans cette mesure.

\section{Références}

\section{Articles scientifiques}

1• J.D. McGraw et al., "Self-similarity and energy dissipation in stepped polymer films", Phys. Rev. Lett. 109 (2012) 128303

2•Y. Chai et al., "A direct quantitative measure of surface mobility in a glassy polymer", Science $\mathbf{3 4 3}$ (2014) 994.

3• T. Salez et al., "Cooperative strings and glassy interfaces", PNAS 112 (2015) 8227.
Articles de presse

- www.lefigaro.fr/sciences/2014/03/05/0100820140305ARTFIG00304-en-surface-certains-verrescoulent-comme-du-miel.php

- www.lemonde.fr/sciences/article/2015/06/29/le-verre -dans-tous-ses-eclats_4664100_1650684.htm

- www.pourlascience.fr/ewb_pages/a/actu-des-chaines -cooperatives-pour-comprendre-la-transition-vitreuse35472.php 